\title{
Morfología comparativa del corión del huevo de Symmetrischema tangolias (Gyen 1913) y Tecia solanivora (Povolny 1973), polillas del cultivo de Solanum tuberosum
}

\section{Comparative morphology of the egg chorion of Symmetrischema tangolias (Gyen 1913) and Tecia solanivora (Povolny 1973), parasitic moths of Solanum tuberosum}

\section{Morfologia comparativa do córion do ovo de Symmetrischema tangolias (Gyen 1913) y Tecia solanivora (Povolny 1973), traças do cultivo de Solanum tuberosum}

\author{
Carolina Martínez G ${ }^{1}$, Carlos A Soria ${ }^{1,2}$, Rubén León $\mathbf{V}^{3}$ \\ ${ }^{1}$ Laboratorio de Bioquímica, Escuela de Ciencias Biológicas, Facultad de Ciencias Exactas y Naturales, Pontificia \\ Universidad Católica del Ecuador, Quito-Ecuador \\ ${ }^{2}$ Laboratorios Labitech Cia. Ltda, Quito-Ecuador. \\ ${ }^{3}$ Grupo WICOM. Universidad de las Fuerzas Armadas. ESPE. Sangolquí. Av. Gral. Rumiñahui s/n. Sangolquí- \\ Ecuador. \\ *drcasp@hotmail.com \\ doi.org/ 10.26807/remcb.v40i1.651
}

Recibido 11-08-2018 ; Aceptado 29-04-2019

\begin{abstract}
RESUMEN. - La técnica de microscopía electrónica de barrido (SEM) fue utilizada para estudiar la estructura externa coriónica de los huevos de la polilla de la papa: Symmetrischema tangolias y Tecia solanivora (Lepidóptera: Gelechiidae). Mediante este estudio, se observó diferencias marcadas en la reticulación de los coriones de ambas especies; se reportó el número de aerópilos y micrópilos por huevo, se describió y diferenció las características superficiales de estas estructuras. Con el uso del microscopio confocal se determinó el tamaño de los ejes mayor y menor, y se propuso un modelo matemático estocástico que identificó a los huevos de cada una de estas especies; usando estos valores se calculó la superficie y el volumen de los huevos de T. solanivora, los cuales resultaron ser más grandes que los de S. tangolias.
\end{abstract}

PALABRAS CLAVES: Corión, Modelo estocástico, Polillas, Symmetrischema tangolias, Tecia solanivora.

ABSTRACT. - Scanning electron microscopy (SEM), was used to study the external chorionic structure of potato moth eggs: Symmetrischema tangolias and Tecia solanivora (Lepidoptera: Gelechiidae). Differences were observed between egg chorium reticulations for each moth species. The number of aeropyls and micropyls per egg were reported and their superficial characteristics were differentiated and described in this study. A confocal microscope was used to determine the size of the major and minor axes of the eggs. These values were used to propose a stochastic mathematical model that identifies each species' eggs, and to calculate their surface area and volume. Results indicate eggs of T. solanivora to be larger than those of S. tangolias.

KEYWORDS: Chorium, Moths, Stochastic model, Symmetrischema tangolias, Tecia solanivora.

RESUMO. - A técnica de Microscopia Electrónica de Varrimento (SEM) foi utilizada para estudar a estrutura externa coriônica dos ovos das traças da papa: Symmetrischema tangolias e Tecia solanivora 
(Lepidóptera: Gelechiidae). Observou-se diferencia marcada na reticulação dos seus córion. Reportou-se o número de aereópilos e micrópilos por ovo, descreveu-se e diferenciou-se as características superficiais das estruturas. Com o microscópio confocal foi determinado o tamanho dos eixes maiores e menores dos ovos, e foi proposto um modelo matemático estocástico que identificou os ovos de cada espécie; com estes valores calculou-se sua superfície e seu volumem. Os ovos de $T$. solanivora resultaram ser maiores que os de $S$. tangolias.

PALAVRAS-CHAVE: Córion, Modelo estocástico, Traças, Symmetrischema tangolias, Tecia solanivora.

\section{INTRODUCCIÓN}

El cultivo de la papa es una actividad importante en varias zonas de los Andes por ser un producto básico en la dieta de sus habitantes. Entre las plagas de este cultivo se encuentra el complejo formado por tres especies de polillas de la familia Gelechiidae del orden Lepidóptera: Phtorimea operculella (Zeller 1873), Symmetrischema tangolias (Gyen 1913) y Tecia solanivora (Povolny 1973) (Kroschel 2002).

Los trabajos realizados en varias plagas del orden Lepidóptera se concentran en estudios de larvas, crisálidas, adultos y sus plantas huéspedes (Hernández et al. 2014a, 2014b) pero hay poca información sobre estructura del endo y exocorión del huevo.

En el corión de los huevos de varios insectos se puede observar regiones especializadas como el micrópilo que permite la entrada del esperma, el hidrópilo que interviene en la captación del agua, el aerópilo para el intercambio gaseoso y en algunas especies se desarrollan diferentes tipos de superficies de fijación como ganchos o dentículos (Gaino et al. 2008, Skudlik et al. 2005, Poprawa y Rost 2004, Simiczyjew 1999, Rosciszewska 1996a, 1996b). El exterior del corión está marcado por una serie de formaciones poligonales que son impresiones de las células foliculares que lo producen (Chávez y Añez 2013, Chapman 1982).

En vista de que algunos de estos aspectos no han sido estudiados en $S$. tangolias y $T$. solanivora, hemos utilizado microscopía electrónica de barrido para caracterizar la morfología externa del corión y reconocer la presencia y número de micrópilos y aerópilos, incluyendo ubicaciones y tamaño de sus diámetros internos. La microscopía confocal fue utilizada para establecer dimensiones estructurales del huevo, acompañado de análisis estadísticos comparativos para demostrar similitudes y diferencias entre estas dos especies. El estudio del corión puede ser otra información importante en la determinación de especies, ya que los huevos y sus estructuras asociadas representan caracteres singulares, útiles en estudios metabólicos o de reconstrucción filogenética (Freitas y Brown 2004, García-Barros y Martin 1995).

\section{MATERIALES Y MÉTODOS}

Las polillas adultas de $S$. tangolias y $T$. solanivora se mantuvieron en condiciones controladas de laboratorio: a la sombra, a $18{ }^{\circ} \mathrm{C}$ promedio, $45 \%$ de humedad, con libre acceso a $1 \mathrm{ml}$ de sucrosa $2 \mathrm{M}$ embebida en una mota de algodón. Se construyeron cámaras de cópula con tubos de $\mathrm{PBC}$ de $30 \mathrm{~cm}$ de largo por $10 \mathrm{~cm}$ de diámetro, se cubrió los extremos del tubo con tela malla ( $2 \mathrm{~mm}$ en cuadro) y cartulina negra al exterior. En cada cámara, colocada en posición vertical, se introdujeron 70 adultos entre machos y hembras; las hembras ovopositaron sujetándose a una de las dos mallas y los huevos terminaron adheridos a una o a ambas cartulinas de los extremos (Mora-Criollo et al. 2013). La recolección de los huevos fue diaria durante nueve días para $S$. tangolias y hasta 11 días para $\mathrm{T}$. solanivora, días que duró la ovoposición.

Para estudiar la morfología externa de los huevos, se utilizó el microscopio electrónico de barrido (modelo SEM JEOL - JSM5310) del Instituto Nacional de Investigación en Salud Pública, INSPI, Guayaquil, Ecuador. Inicialmente se usaron 10 huevos correspondientes al sexto día de postura, fijados por 12 horas o durante una semana en glutaraldehído al $3 \%$, y en formalina al $80 \%$ preparada de concentraciones comerciales que viene al $33 \%$. En una siguiente metodología se utilizó 10 huevos frescos no fijados, por especie, igualmente del sexto día de incubación, los mismos que fueron recubiertos automáticamente con una capa fina conductiva de oro en una cámara automática Fine Coater JEOL JFC-1200, previo al barrido electrónico durante la toma de imágenes con el microscopio SEM JEOL.

La técnica microscópica permitió fotografiar la estructura externa del corión, contar el número de 
aerópilos por campo en cinco campos microscópicos al azar por huevo. El número promedio de aerópilos en cada huevo fue relacionado a la superficie total promedio de cada uno de cinco huevos observados, reportándose la mediana del número de aerópilos por huevo; igualmente se calculó la mediana del diámetro interno de estos aerópilos en cinco huevos observados por especie, y en la búsqueda de al menos un micrópilo por huevo se observaron hasta 25 campos diferentes en cada uno de los huevos por especie. La prueba de Mann Whitney fue utilizada para establecer semejanzas o diferencias significativas entre las medianas del número y diámetro interno de los aerópilos en las dos especies (Martínez 2015).

Para estudiar superficie, volumen, forma y tamaño de los huevos, se utilizó el microscopio confocal (Olympus FV100) del Laboratorio de Fitopatología de la Agencia Ecuatoriana de Aseguramiento de Calidad del Agro, Agrocalidad, Tumbaco, Ecuador. Inicialmente se procedió a fijar los huevos con paraformaldehído al $4 \%$ durante 10 minutos, previo a las observaciones microscópicas. Seguidamente se ensayó otra metodología con huevos frescos igualmente colectados durante el día requerido después de la ovoposición, los mismos que fueron teñidos durante cinco minutos con el cromatóforo fluorescente RH414 (Invitrogen) diluido con buffer fosfato $0,5 \mathrm{M}, \mathrm{pH} 7$ en una relación de $1: 50 \mu \mathrm{l}$. La muestra teñida fue lavada tres veces con $50 \mu 1$ buffer carbonato $0,5 \mathrm{M}, \mathrm{pH} 10$, dejando un minuto entre cada lavada.

En un portaobjetos de vidrio se depositaron tres gotas de glicerina y sobre cada una de ellas se colocaron 20 huevos teñidos, 60 en total, colectados de una misma especie, en cada uno de los días del ciclo de maduración del huevo; de estos 60 huevos se seleccionaron aleatoriamente 25 de ellos los cuales mantuvieron intactas sus delicadas estructuras. Con el Software FV10-ASW 3.0 del microscopio confocal se tomaron medidas del eje mayor y del eje menor. Se calculó la superficie total y el volumen de cada huevo usando las fórmulas que corresponden a las de un elipsoide. Se estudiaron en total 225 huevos de $S$. tangolias, 25 por cada día de los nueve que tardaron en eclosionar y 275 de $T$. solanivora porque estos eclosionaron a los 11 días después de la ovoposición.

El software SPSS versión 18.0 fue utilizado para el análisis estadístico de los ejes mayor y menor, el cálculo de la superficie del corión y el volumen total del huevo de cada especie, para cada día de maduración previo a la eclosión. Se utilizó la prueba de Kolmogorov-Smirnov (Sánchez-Otero 2015) para comprobar la normalidad de los datos de los ejes. Se realizó el análisis de varianza (ANOVA) para establecer diferencias significativas entre los días de maduración de los huevos antes de la eclosión y la muestra de huevos por día de recolección, respecto a los ejes mayor y menor de los huevos. Se estimaron los componentes añadidos de varianza y se observó la magnitud de la variación atribuible a los diferentes componentes del modelo utilizado (diseño completamente aleatorio). Se aplicó la prueba del Estudiante para establecer las diferencias entre el tamaño de los huevos de cada especie sobre la variable superficie y la homogeneidad de las varianzas de los ejes a través de la prueba de Levene (Sánchez-Otero 2015).

Se aplicó un modelo matemático estocástico para estimar posibles diferencias o similitudes en dos ejes (largo y ancho) de los huevos de las dos especies: el eje mayor o variable aleatoria $X$ y el eje menor o variable aleatoria Y en 225 huevos de S. tangolias y 275 huevos de $T$. solanivora.

El resultado del modelo matemático se contrastaría con las medidas reales de los ejes.

\section{RESULTADOS}

La fijación de los huevos de polilla con técnicas convencionales a base de formaldehído, paraformaldehído o glutaraldehído, alasconcentraciones y tiempos indicados, no pudieron ser utilizadas porque ocasionaban pérdida del detalle geométrico del corión incluyendo la reducción del tamaño del huevo.

El análisis de los huevos frescos recubiertos con una capa fina de oro para los estudios de microscopía electrónica o teñidos con el cromatóforo RH414 para la microscopía confocal, nos permitieron observar formas, tamaños y estructuras características del huevo de cada especie.

El eje mayor del huevo de S. tangolias (Fig. 1, Tabla 1,3) presentó una media de 0,47 $\pm 0,04 \mathrm{~mm}$ y su eje menor de $0,35 \pm 0,03 \mathrm{~mm}$, la relación largo: ancho fue de 1,29. La media de la superficie externa fue de $0,49 \pm 0,07 \mathrm{~mm} 2 \mathrm{y}$ su volumen de $0,03 \pm 0,01 \mathrm{~mm} 3$.

Los huevos de $T$. solanivora presentaron un eje mayor medio de $0,53 \pm 0,05 \mathrm{~mm}$ y un eje menor medio de $0,41 \pm 0,05 \mathrm{~mm}$ con una relación largo: 
Tabla 1. Estadísticos descriptivos1 de las variables estudiadas en Symmetrischema tangolias $(\mathrm{n}=225)$

\begin{tabular}{|c|c|c|c|c|c|c|c|}
\hline & \multirow{2}{*}{$\begin{array}{l}\text { Media } \\
(\mathrm{mm})\end{array}$} & \multirow{2}{*}{$\begin{array}{l}\text { Desviación } \\
\text { típica } \\
\text { (mm) }\end{array}$} & \multirow{2}{*}{$\begin{array}{l}\text { Error } \\
\text { típico } \\
(\mathrm{mm})\end{array}$} & \multicolumn{2}{|c|}{$\begin{array}{c}\text { Intervalo de } \\
\text { confianza para la } \\
\text { media al 95\% }\end{array}$} & \multirow{2}{*}{$\begin{array}{c}\text { Mínimo } \\
(\mathrm{mm})\end{array}$} & \multirow{2}{*}{$\begin{array}{r}\text { Máximo } \\
(\mathrm{mm})\end{array}$} \\
\hline & & & & $\begin{array}{c}\text { Límite } \\
\text { inferior } \\
(\mathrm{mm})\end{array}$ & $\begin{array}{l}\text { Límite } \\
\text { superior } \\
\text { (mm) }\end{array}$ & & \\
\hline Eje mayor & 0,47 & 0,04 & 0,002 & 0,47 & 0,48 & 0,35 & 0,57 \\
\hline Eje menor & 0,35 & 0,03 & 0,002 & 0,35 & 0,36 & 0,27 & 0,48 \\
\hline Superficie & 0,49 & 0,07 & 0,005 & 0,48 & 0,5 & 0,31 & 0,71 \\
\hline Volumen & 0,03 & 0,01 & 0,001 & 0,03 & 0,03 & 0,02 & 0,06 \\
\hline $\begin{array}{l}\text { Superficie/ } \\
\text { Volumen }\end{array}$ & 15,74 & 1,27 & 0,08 & 15,58 & 15,91 & 12,62 & 19,73 \\
\hline
\end{tabular}

${ }^{1}$ Datos del análisis descriptivo realizado con el Software SPSS versión 18.0
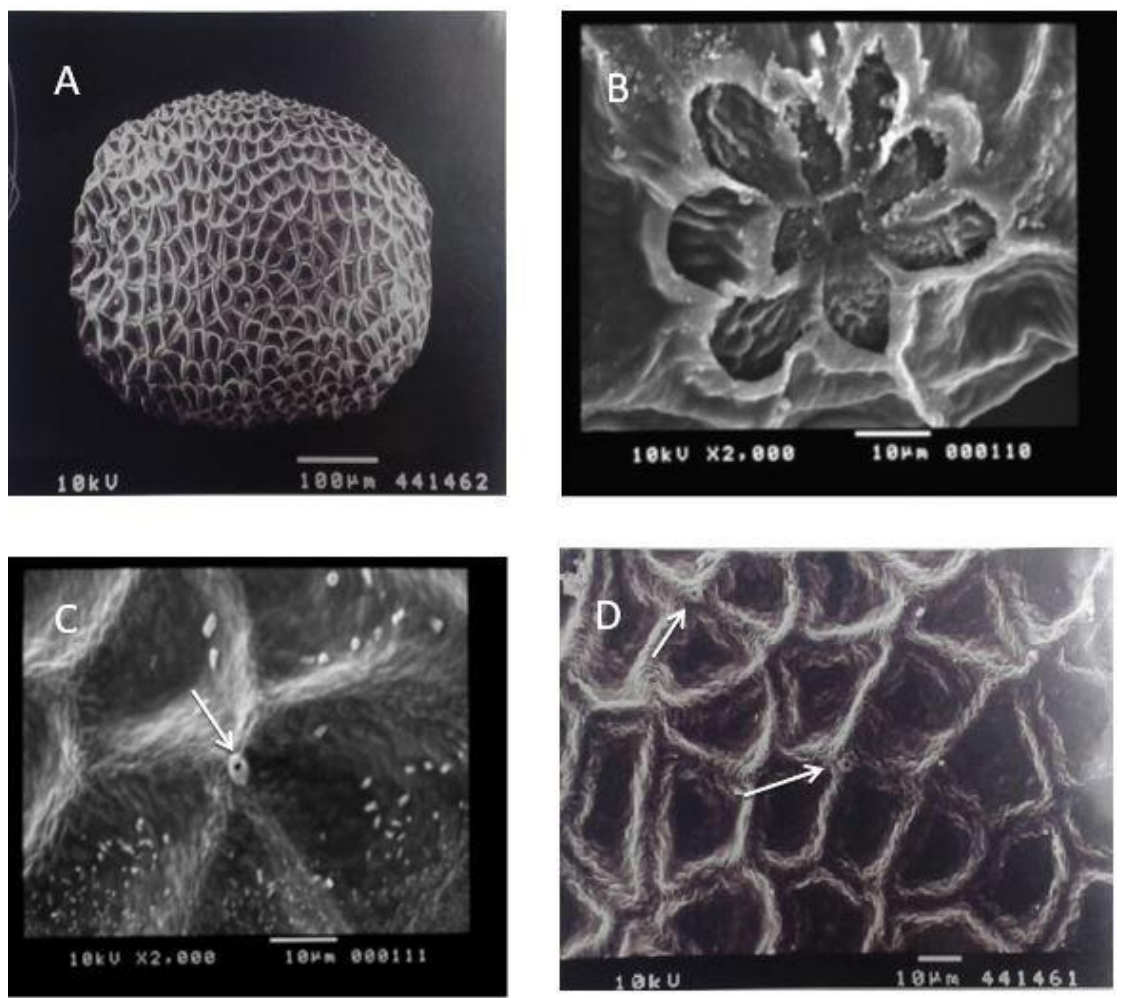

Figura 1, Huevo de Symmetrischema tangolias: microscopía electrónica de barrido, (A) Reticulación del corión, (B) Micrópilo: se observa la roseta principal, (C, D) Aerópilos en la convergencia de los polígonos del corión. 
ancho de 1,34. La mediana de la superficie externa del huevo fue de 0,63 $\pm 0,09 \mathrm{~mm} 2 \mathrm{y}$ un volumen de $0,05 \pm 0,01 \mathrm{~mm} 3$.

El exocorión de $S$. tangolias tuvo apariencia más rugosa que el de $T$. solanivora cuya superficie se ve más homogénea y aparentemente más delicada. En ambas especies se encontró en
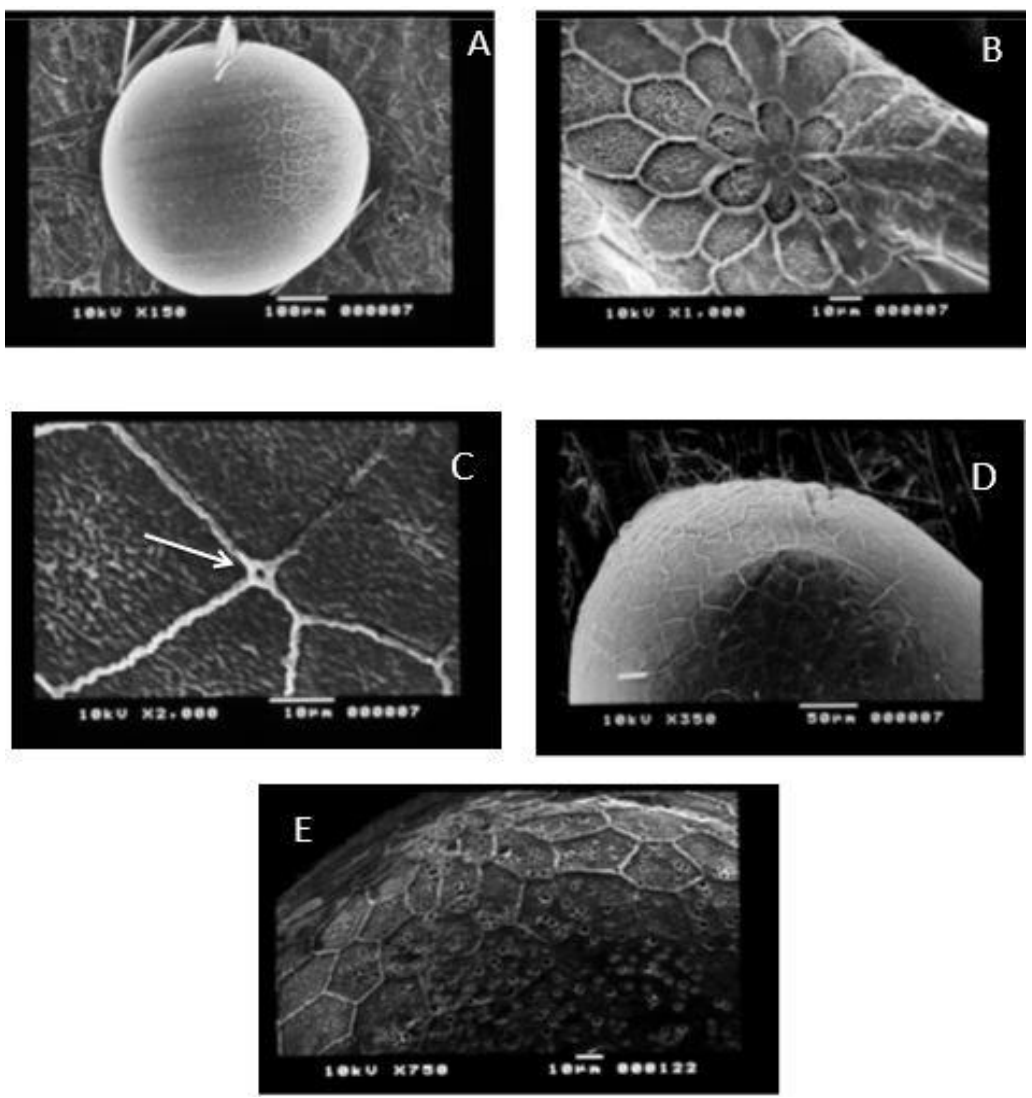

Figura 2, Huevo de Tecia solanivora: microscopía electrónica de barrido, (A) Reticulación del corión, (B) Micrópilo: se observa la roseta principal (interior) y secundaria (exterior), (C) Aerópilo en la convergencia de los polígonos del corión, (D) Reticulación (E) Presencia de erosiones en la superficie del corión.

Tabla 2. Estadísticos descriptivos1 de las variables estudiadas en Tecia solanivora $(\mathrm{n}=275)$

\begin{tabular}{|c|c|c|}
\hline Característica & Symmetrischema tangolias & $\begin{array}{c}\text { Tecia } \\
\text { solanivora }\end{array}$ \\
\hline Eje Mayor (mm) & $0,47 \pm 0,04$ & $0,53 \pm 0,05$ \\
\hline Eje Menor (mm) & $0,35 \pm 0,03$ & $0,41 \pm 0,04$ \\
\hline $\begin{array}{l}\text { Relación Eje mayor/ } \\
\text { Eje menor }\end{array}$ & 1,29 & 1,34 \\
\hline Superficie $\left(\mathrm{mm}^{2}\right)$ & $0,49 \pm 0,07$ & $0,63 \pm 0,09$ \\
\hline Volumen $\left(\mathrm{mm}^{3}\right)$ & $0,03 \pm 0,01$ & $0,05 \pm 0,01$ \\
\hline Relación S:V & $15,74 \pm 1,27$ & $13,88 \pm 1,2$ \\
\hline \# de aerópilos ** & 10,2 & 25,4 \\
\hline $\begin{array}{l}\text { Diámetro del aerópilo } \\
(\mu \mathrm{m}) *\end{array}$ & 1 & 1,2 \\
\hline \# de Micrópilos* & 1 & 1 \\
\hline $\begin{array}{l}\text { Diámetro de micrópilo } \\
(\mu \mathrm{m})^{*}\end{array}$ & 3,8 & 4,6 \\
\hline
\end{tabular}

Los datos corresponden al promedio más / menos la desviación estándar,

*E1 dato corresponde a la mediana,
**Promedio de aerópilos de cada especie dividido para la superfície promedio de los huevos. 
cada huevo solo un micrópilo ubicado en el polo anterior. El diámetro interno del micrópilo de $S$. tangolias presentó una mediana de $3,8 \mu \mathrm{m}$ (Tabla 3) el mismo que estuvo rodeado de una roseta o conjunto de impresiones células como de cinco a siete pétalos (Fig. 1B). El diámetro del micrópilo de $T$. solanivora midió $4,6 \mu \mathrm{m}$, su roseta principal consistió de siete impresiones celulares o pétalos la misma que a su vez aparecía rodeada o protegida de otra secundaria en la que se contaron cuatro o 15 pétalos (Fig. 2B).

En los huevos estudiados de cada especie se observaron aerópilos unitariamente localizados en algunos vértices donde convergían radiales poligonales que formaban una especie de anillo celular. Los aerópilos de $S$. tangolias tenían un diámetro interno con una mediana de $1 \mu \mathrm{m} \quad \mathrm{y}$ su número reportado para esta especie fue de una mediana de 10 aerópilos por huevo (Tabla 3, Figs.1C, D). En T. solanivora se observó que los aerópilos tenían un diámetro con una mediana de $1,2 \mu \mathrm{m}$ y una mediana de 25 aerópilos por huevo (Tabla 3, Figs. 2C, D). En esta especie, se observó gran cantidad de depresiones, lagunas u orificios, en toda la superficie del exocorión, los cuales no fueron encontrados en los huevos de $S$. tangolias. Se detectaron diferencias significativas en la variación de las medidas de los huevos conforme avanzó el período de incubación para ambas especies, sin embargo, estas variaciones no mostraron un aumento de tamaño en forma monotónica, sino más bien en forma irregular. Para explicar estas variaciones se calcularon los componentes añadidos de varianza, sobre la variable superficie del corión, demostrando que se produjo una fuerte heterogeneidad entre los huevos, antes que entre los días de incubación: para $T$. solanivora solo el 4,3 $\%$ de la variación ocurrió entre los días, en tanto que el 95,7 \% de variación ocurrió entre los huevos, dentro de los días de maduración. Con S. tangolias el 9,1\% de la variación ocurrió entre los días y el $89,9 \%$ entre los huevos, dentro de los días previos a la eclosión.

Las Tablas 1,2 y 3 reportan diferencias altamente significativas $(\mathrm{t}=-18,88, \mathrm{gl}=492, \mathrm{p}=0,001)$ entre los huevos de T. solanivora que son más grandes $\left(\mathrm{X}^{-}=0,63 \pm 0,1 \mathrm{~mm}^{\wedge} 2\right)$, comparados con los de

$S$. tangolias $\left(\mathrm{X}^{-}=0,49 \pm 0,07 \mathrm{~mm}^{\wedge} 2\right)$. En el antisde los aerópilos (Tabla 3), se tomó en cuenta los estadísticos mediana e IQR (Rango Interquartil), debido al número de la muestra y a la distribución asimétrica que presentaron, lo que dio como resultado los valores de mediana 10,24 e IQR 13,27 en los huevos de S.tangolias y mediana 25,4 e

Tabla 3. Parámetros comparativos y características de los huevos de dos especies de polillas

\begin{tabular}{|c|c|c|}
\hline Característica & Symmetrischema tangolias & $\begin{array}{c}\text { Tecia } \\
\text { solanivora }\end{array}$ \\
\hline Eje Mayor (mm) & $0,47 \pm 0,04$ & $0,53 \pm 0,05$ \\
\hline Eje Menor (mm) & $0,35 \pm 0,03$ & $0,41 \pm 0,04$ \\
\hline $\begin{array}{l}\text { Relación Eje mayor/ } \\
\text { Eje menor }\end{array}$ & 1,29 & 1,34 \\
\hline Superficie $\left(\mathrm{mm}^{2}\right)$ & $0,49 \pm 0,07$ & $0,63 \pm 0,09$ \\
\hline Volumen $\left(\mathrm{mm}^{3}\right)$ & $0,03 \pm 0,01$ & $0,05 \pm 0,01$ \\
\hline Relación S:V & $15,74 \pm 1,27$ & $13,88 \pm 1,2$ \\
\hline \# de aerópilos ** & 10,2 & 25,4 \\
\hline $\begin{array}{l}\text { Diámetro del aerópilo } \\
(\mu \mathrm{m}) *\end{array}$ & 1 & 1,2 \\
\hline \# de Micrópilos* & 1 & 1 \\
\hline $\begin{array}{l}\text { Diámetro de micrópilo } \\
(\mu \mathrm{m})^{*}\end{array}$ & 3,8 & 4,6 \\
\hline
\end{tabular}

Los datos corresponden al promedio más / menos la desviación estándar,

*E1 dato corresponde a la mediana,

**Promedio de aerópilos de cada especie dividido para la superficie promedio de los huevos. 
IQR 3,97 para T. solanivora. Se realizó la prueba de Mann-Whitney U para comparar el número de aerópilos en los huevos de cada especie $(\mathrm{p}=0,008)$.

El número de aerópilos por $\mathrm{mm} 2$ de superficie de corión fue mayor en $T$. solanivora $(40,3)$, en contraste con $S$. tangolias $(20,8)$. De igual forma, la mediana del diámetro interno de estos aerópilos en $T$. solanivora fue de $1,2 \mu \mathrm{m}$ y de $1 \mu \mathrm{m}$, en S. tangolias $(\mathrm{p}=0,003)$.

La distribución de las variables X y Y se ajustaron convenientemente a la distribución Gaussiana. Se seleccionó el modelo Gaussiano por su simplicidad pues bastaron los dos primeros momentos para que las variables aleatorias de los ejes X y Y queden completamente caracterizadas (Tabla 4, Fig. 3).
Desde el punto de vista estocástico, los ejes mayor y menor quedaron caracterizados por las siguientes funciones densidad de probabilidad Gaussianas:

Para Symmetrischema tangolias:

$$
f_{X 1}\left(x_{1}\right)=\frac{1}{\sigma_{x 1} \sqrt{2 \pi}} \exp \left[\frac{-\left(X_{1}-m_{x 1}\right)^{2}}{2 \sigma_{x 1}^{2}}\right]=\frac{1}{0,036 \sqrt{2 \pi}} \exp \left[\frac{-\left(X_{1}-0,47\right)^{2}}{2(0,001296)}\right]
$$

donde:

$\mathrm{X}_{1}$ : Longitud del eje mayor de S. tangolias. $\mathrm{m}_{\mathrm{X} 1}$ : Media del eje mayor de $S$. tangolias. $\sigma_{\mathrm{x} 1}$ : Desviación estándar de eje mayor de $S$. tangolias.

$$
f_{Y 1}\left(y_{1}\right)=\frac{1}{\sigma_{y 1} \sqrt{2 \pi}} \exp \left[\frac{-\left(Y_{1}-m_{y 1}\right)^{2}}{2 \sigma_{y 1}^{2}}\right]=\frac{1}{0,034 \sqrt{2 \pi}} \exp \left[\frac{-\left(Y_{1}-0,35\right)^{2}}{2(0,001156)}\right]
$$

Tabla 4, Medias (mm) y varianzas $(\sigma)$ de los ejes mayor y menor de los huevos de dos polillas

\begin{tabular}{lcc}
\hline & Symmetrischema & \\
Característica & tangolias & Tecia solanivora \\
& $\mathbf{X}_{\mathbf{1}}, \mathbf{Y}_{\mathbf{1}}$ & $\mathbf{X}_{2}, \mathbf{Y}_{\mathbf{2}}$ \\
\hline Eje mayor (mm) & & \\
$\mathbf{X}$ & $\mathrm{mx} 1=0,47$ & $\mathrm{mx}_{2}=0,53$ \\
& $\sigma \times 1=0,036$ & $\sigma \times 2=0,049$ \\
Eje menor (mm) & & \\
$\mathbf{Y}$ & $\mathrm{mY} 1=0,35$ & $\mathrm{~m}_{\mathrm{Y} 2}=0,41$ \\
& $\sigma \mathrm{Y} 1=0,034$ & $\sigma \mathrm{Y} 2=0,045$ \\
\hline
\end{tabular}

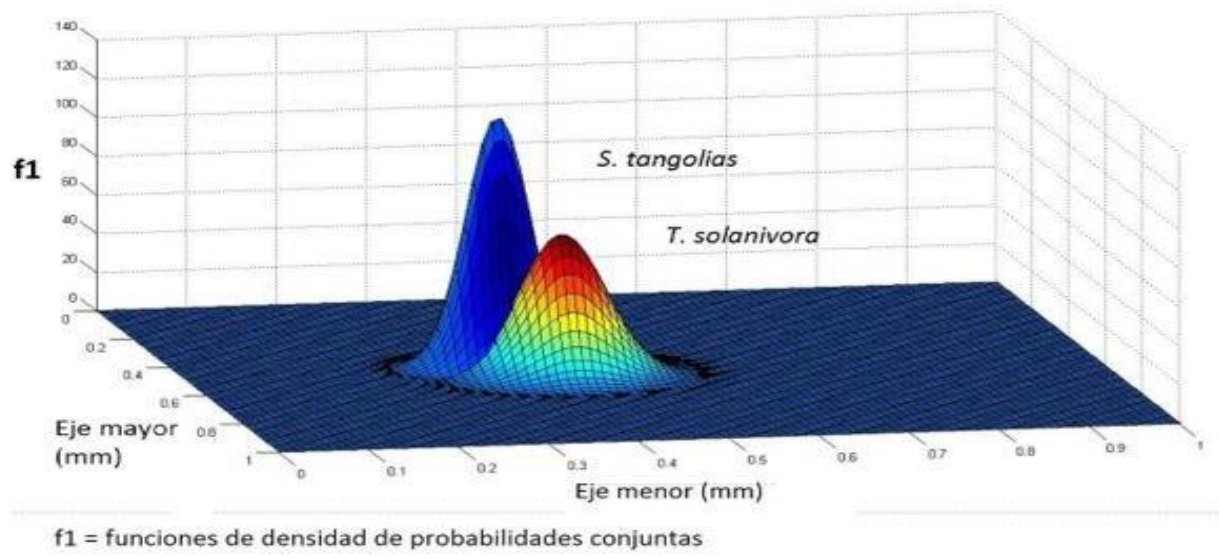

Figura 3, Funciones (f1) de densidad de probabilidades conjuntas de los ejes mayor y menor correspondiente a Symmetrischema tangolias y a Tecia solanivora, Gráfico realizado en el Software Mathlab. 
donde:

$\mathrm{Y}_{1}$ : Longitud del eje menor de $S$. tangolias.

$\mathrm{m}_{\mathrm{Y} 1}$ : Media del eje menor de $S$. tangolias.

$\sigma_{\mathrm{Y} 1}$ : Desviación estándar de eje menor de

S. tangolias.

Para Tecia solanivora

$f_{X 2}\left(x_{2}\right)=\frac{1}{\sigma_{x 2} \sqrt{2 \pi}} \exp \left[\frac{-\left(X_{2}-m_{x 2}\right)^{2}}{2 \sigma_{x 2}^{2}}\right]=\frac{1}{0,049 \sqrt{2 \pi}} \exp \left[\frac{-\left(X_{2}-0,53\right)^{2}}{2(0,002401)}\right]$

$\mathrm{X}_{2}$ : Longitud del eje mayor de $T$. solanivora.

$\mathrm{m}_{\mathrm{X} 2}$ : Media del eje mayor de T. solanivora.

$\sigma_{\mathrm{X} 2}$ : Desviación estándar de eje mayor de

T. solanivora.

$f_{Y 2}\left(y_{2}\right)=\frac{1}{\sigma_{y 2} \sqrt{2 \pi}} \exp \left[\frac{-\left(Y_{2}-m_{y 2}\right)^{2}}{2 \sigma_{y 2}^{2}}\right]=\frac{1}{0,045 \sqrt{2 \pi}} \exp \left[\frac{-\left(Y_{2}-0,41\right)^{2}}{2(0,002025)}\right]$

donde:

$\mathrm{Y}_{2}$ : Longitud del eje menor de T. solanivora. $\mathrm{m}_{\mathrm{Y} 2}$ : Media del eje menor de T. solanivora. $\sigma_{\mathrm{Y} 2}$ : Desviación estándar de eje menor de T. solanivora.

Las expresiones anteriormente obtenidas mostraron que las desviaciones estándar de los ejes mayor y menor de cada especie fueron muy similares (S. tangolias $\mathrm{p}=0,562$ y $T$. solanivora $\mathrm{p}=0,977$ ); las desviaciones del eje mayor y del eje menor de $S$. tangolias fueron $0,036 \mathrm{~mm}$ y $0,034 \mathrm{~mm}$ respectivamente, y de $T$. solanivora $0,049 \mathrm{~mm}$ y $0,045 \mathrm{~mm}$ (Tabla 4). Por simplicidad matemática y para su uso en el modelo predictivo se obtuvo un valor medio de estas desviaciones: $0,035 \mathrm{~mm}$ para S. tangolias y $0,047 \mathrm{~mm}$ para $T$. solanivora.

La caracterización anteriormente descrita para los ejes mayor y menor, sirvió para que a través de la prueba de hipótesis binaria (Procesador de la Razón de Verosimilitud) la cual minimiza el llamado riesgo de Bayes (Van Trees et al. 2013), se pueda determinar a qué especie de la polilla de la papa pertenecería un huevo con dimensiones $X$ y Y. Es posible demostrar que el logaritmo natural de la razón de verosimilitud considerando X y Y variables aleatorias Gaussianas independientes y la hipótesis $\mathrm{H}_{1}=T$. solanivora y $\mathrm{H}_{0}=S$. tangolias, vendría dado por la siguiente expresión:
Razón de Verosimilitud (Van Trees et al. 2013):

$$
\bigwedge(\underline{r})=\frac{f_{\underline{R}} / H_{1}\left(\underline{r} / H_{1}\right)}{f_{\underline{R}} / H_{\mathrm{O}}\left(\underline{r} / H_{0}\right)} \underset{H^{0}}{\stackrel{H^{1}}{>}} \gamma
$$

En el caso $\mathrm{H}_{1}$, bajo la consideración de que X y $\mathrm{Y}$ son independientes, el numerador de la expresión vendría dado por:

$$
f_{X_{2} Y_{2}\left(x_{2}, y_{2}\right)=} f_{X_{2}\left(x_{2}\right)} f_{Y_{2}\left(y_{2}\right)}
$$

donde:

X2: Longitud del eje mayor de $T$. solanivora con $\mathrm{m}_{\mathrm{x} 2}=0.53$ y $\sigma_{\mathrm{x} 2}=0.047$

Y2: Longitud del eje menor de T. solanivora con $\mathrm{m}_{\mathrm{Y} 2}=0.41$ y $\sigma_{\mathrm{Y} 2}=0.047$

Dando como resultado:

$$
f_{X_{2} Y_{2}\left(x_{2}, y_{2}\right)=} \frac{1}{0,047 \sqrt{2 \pi}} \exp \left[\frac{-\left(X_{2}-0,53\right)^{2}}{2(0.047)^{2}}\right] \cdot \frac{1}{0,047 \sqrt{2 \pi}} \exp \left[\frac{-\left(Y_{2}-0,41\right)^{2}}{2(0,047)^{2}}\right]
$$

En el caso $\mathrm{H}_{0}$ bajo la consideración de que X y Y son independientes, el denominador de la expresión vendría dado por:

$$
f_{X_{1} Y_{1}\left(x_{1}, y_{1}\right)=} f_{X_{1}\left(x_{1}\right)} f_{Y_{1}}\left(y_{1}\right)
$$

donde:

$\mathrm{X} 1$ : Longitud del eje mayor de $S$. tangolias $\operatorname{con} \mathrm{m}_{\mathrm{X} 1}$ $=0,47$ y $\sigma_{x 1}=0,035$

Y1: Longitud del eje menor de $S$. tangolias con $\mathrm{m}_{\mathrm{Y} 1}$ $=0,35$ y $\sigma_{\mathrm{Y} 1}=0,035$

Dando como resultado:

$f_{X_{1} Y_{1}\left(x_{1}, y_{1}\right)=} \frac{1}{,, 035 \sqrt{2 \pi}} \exp \left[\frac{-\left(X_{1}-0,47\right)^{2}}{2(0,035)^{2}}\right] \cdot \frac{1}{0,035 \sqrt{2 \pi}} \exp \left[\frac{-\left(Y_{1}-0,35\right)^{2}}{2(, .035)^{2}}\right]$

Por tanto el $\operatorname{Ln}[\bigwedge(\underline{r})] \quad$ se reduce a:

$$
\operatorname{Ln}[.]=\operatorname{Ln}\left[f_{X_{2}\left(x_{2}\right)} f_{Y_{2}\left(y_{2}\right)}\right]-\operatorname{Ln}\left[f_{X_{1}\left(x_{1}\right)} f_{Y_{1}\left(y_{1}\right)}\right]
$$

Sustituyendo las expresiones $f_{X_{1}\left(x_{1}\right), f f_{Y_{1}}\left(y_{1}\right), f_{X_{2}}\left(x_{2}\right), f_{Y_{2}}\left(y_{2}\right)}$ anteriormente determinadas y reordenando los términos, se obtuvo la siguiente expresión: 


$$
\operatorname{Ln}\left(\frac{0.035^{2}}{0,047^{2}}\right)+\frac{(X-0,47)^{2}+(Y-0,35)^{2}}{2(0,035)^{2}}-\frac{(X-0,53)^{2}+(Y-0,41)^{2}}{2(0,047)^{2}} \underset{H 0}{>} 0
$$

donde:

$\mathrm{X}$ : Longitud del eje mayor.

Y: Longitud del eje menor.

Utilizando la fórmula descrita se obtuvo un 86,2 $\%$ de aciertos en el caso de $S$. tangolias y un 84 $\%$ de aciertos para T. solanivora, según el modelo estadístico propuesto (Tabla 5).

\section{DISCUSIÓN}

Se ha estudiado algunos aspectos de la morfología externa del corión de los huevos de dos especies de polillas de la papa: $S$. tangolias y $T$. solanivora, en cuanto a la forma, reticulación, dimensiones, relación superficie: volumen, presencia y número de aerópilos y de micrópilos.

En estudios de lepidóptera se ha reportado huevos elipsoides, fusiformes, oblongados o alargados (Hernández et al. 2014a, 2014b); en la familia Gelechiidae también reportaron huevos fusiformes (Arbogast et al. 1980) mientrasqueen Nymphalidae, Pieridae, Saturniidae y Piralidae, donde se han hecho la mayoría de estudios, se encontraron varias formas incluyendo las elipsoideas (Llorente y Castro 2007, Kawaguchi et al. 2007, García-Barros y Martín 1995, Arbogast et al. 1980).

En nuestro estudio se encontró que los huevos de estas dos especies, si bien se les considera a ambos como elipsoides (Figs. 1A, 2A), si son diferentes ya que los de T. solanivora son de mayor tamaño y más redondeados porque la relación calculada entre los ejes mayor y menor, es menor, comparado con lo que se encontró para $S$. tangolias, que son francamente elipsoides. Las variaciones observadas en cuanto al tamaño de los huevos en relación a los días de maduración, fueron atribuidas a la heterogeneidad del tamaño de los huevos colectados en diferentes días. Se entiende que ocurren cambios estructurales moleculares en la superficie del huevo a medida que se acerca el tiempo de eclosión.

El corión de los huevos de T.solanivora y S.tangolias muestran diferentes patrones de reticulación, característica que permite su diferenciación. Esta diferencia podría deberse a que la capa lipoproteica que se adhiere al huevo, mientras se forma el exocorión, se concentra en los bordes celulares (Chapman 1982). Y debido a que la impresión reticular en $S$. tangolias es notoriamente más marcada que en $T$. solanivora (Figs. 1C, 2C), se podría inferir que las secreciones son más pronunciadas y abundantes en la primera, incluso la distribución y forma de los polígonos en el corión, es claramente diferente en las dos especies Figs. 1D, 2D).

Impresiones poligonalesenlas superficies coriónicas de los huevos parecen ser comunes; se ha reportado en géneros muy diferentes de Lepidóptera como Spodoptera sp. o Samia sp. (Kawaguchi et al. 2007), incluso en diferentes órdenes, en los que se describe impresiones desde pentagonales hasta octagonales

Tabla 5, Porcentaje de clasificación correcta del número $(\mathrm{N})$ de muestras de polillas analizadas en base al modelo estadístico propuesto

\begin{tabular}{|c|c|c|c|}
\hline \multicolumn{4}{|l|}{ Especie } \\
\hline \multicolumn{2}{|c|}{ Symmetrischema tangolias } & \multicolumn{2}{|c|}{ Tecia solanivora } \\
\hline $\mathbf{N}=$ & $\%$ de $\mathrm{N}$ & $\mathbf{N}=$ & $\%$ de $\mathrm{N}$ \\
\hline $194^{*}$ & $86,20^{*}$ & $231^{*}$ & $84,00^{*}$ \\
\hline $31^{* * *}$ & $13,80^{* * *}$ & $44^{* * *}$ & $16,00^{\text {*** }}$ \\
\hline 225 & 100 & 275 & 100 \\
\hline
\end{tabular}


(Visciarelli et al. 2004, Chávez y Añez 2003,). Sin embargo, deben existir características únicas entre especies, como lo que se ha encontrado, entre S. tangolias (polígonos muy variados) y

T. solanivora (polígonos muy uniformes).

El número de aperturas micropilares es característica de cada especie de insectos (Skudlik et al. 2005). El orden Lepidóptera, por ejemplo, presenta entre uno a 20 micrópilos por huevo (Hinton 1981) a través de los cuales ingresa el esperma para fertilizar al huevo (Deep y Rose 2014, Kučerová et al, 2010, Cruz-Reyez y Camargo-Camargo 2001, Chapman 1982) al momento del desove. Celdas primarias ubicadas como rosetas de pétalos, rodean a los poros micropilares (Suledere 1988) las mismas que definen, identifican y/o protegen el orificio micropilar.

El número de pétalos al igual que el número de rosetas pueden variar en los huevos de Lepidóptera (García-Barros y Martin 1995), su diseño y el número de los mismos garantizan la conservación de la especie (Arbogast 1980). Otros estudios podrían explicar las diferencias estructurales que se reportan en el único micrópilo encontrado en cada uno de los huevos de ambas especies (Figs. 1B, 2B). El tamaño de su diámetro interno y las diferencias en las rosetas que le acompañan tendrá que ver con especificidad, atracción y entrada de los espermatozoides al huevo de cada especie.

Los aerópilos son pórticos que se abren o se cierran, a través de los cuales, los huevos de los insectos realizan el intercambio gaseoso (Kučerová et al, 2010, Cummings y King 1996, Chapman 1982, Tuft 1950). En Lepidóptera, los aerópilos no siempre se encuentran presentes, y cuando los hay, estos pueden variar en su ubicación, tamaño, número y forma: el borde externo de los aerópilos puede ser o no especializado y puede mostrarse bien desarrollado o pobremente definido (Arbogast et al. 1980). En los huevos de las dos especies estudiadas, se observó la presencia asimétrica de aerópilos ubicados en algunos vértices de los polígonos del corión (Figs. 1C, 2C); se encontró que T. solanivora poseía más aerópilos por huevo y por $\mathrm{mm} 2$ de corión, con diámetros internos más amplios que los encontrados en S. tangolias (Tabla 3; Figs 1C, 2C).

Tecia solanivora proviene de zonas relativamente templadas de América Central y de la parte norte de América del Sur, a diferencia de S. tangolias que es más universal (Sporleder et al, 2016), es más pequeña, tiene un ciclo de vida más corto y puede habitar incluso zonas más altas con temperaturas más bajas (Dangles et al, 2008) y cuotas bajas de oxígeno. Se esperaría que la carga metabólica del huevo de $T$. solanivora sea mayor a la de $\mathrm{S}$. tangolias, habrá, por lo tanto, una mayor exigencia de oxígeno e intercambio gaseoso debido a su mayor tamaño, un mayor número de aerópilos por área de corión con diámetros significativamente más amplios y barreras reticulares superficiales más delgadas, lo cual favorecería el flujo, el volumen y el intercambio gaseoso que es facilitado por la forma casi redonda de este huevo.

Es motivo de interés la presencia de una cantidad homogénea y considerable de pequeñas hendiduras, probablemente orificios, cuyo propósito desconocemos y que están ausentes en la otra especie, a pesar de que ambos fueron sometidos a tratamientos similares previos a la observación. Entender el mecanismo de difusión de gases y metabolitos a través del corión (Outram 1967, Tuft 1950) permitiría el uso de ovicidas (Gautam et al. 2015, 2014) en formulaciones capaces de penetrar por estos pórticos y afectar a la larva antes de la eclosión a través de diferentes mecanismos bioquímicos de control.

Desde otro ángulo, la estructura más intrincada que se observa en los huevos de $S$. tangolias podría protegerlos mejor y adaptarlos a un metabolismo más eficiente pero menos exigente respecto a las limitadas cuotas de oxígeno de las zonas altas a las que los huevos se adaptarían mejor.

Las diferencias morfológicas del corión, el tamaño y la forma de los huevos, el número de los aerópilos, la estructura de los micrópilos y aerópilos, permite distinguirlos con facilidad. Es más, el modelo matemático desarrollado, considerando funciones de densidad probabilísticas aleatorias Gaussianas, más la prueba de la hipótesis binaria o razón de verosimilitud, también ayuda a identificar a los huevos de la una o de la otra especie (Fig. 3) con altos grados de confiabilidad.

\section{AGRADECIMIENTOS}

Al Laboratorio de Bioquímica de la Escuela de Ciencias Biológicas, Facultad de Ciencias

Exactas y Naturales de la PUCE; al laboratorio de

Microscopía Electrónica del INSPI, Guayaquil;

al Laboratorio de Fitopatología de Agrocalidad,

Tumbaco por el uso del microscopio Confocal;

a Clifford Keil, Julio Sánchez, Sabrina Méndez, Linda Díaz y Yasiju Anano por el 
apoyo técnico recibido durante algunas fases de este estudio; al Ministerio del Ambiente por otorgar el Permiso de Colección 003-17-IC-FAU-DNB/ MA ; a la PUCE por los fondos destinados al proyecto de investigación N13349: Biomoléculas potencialmente activas.

\section{REFERENCIAS BIBLIOGRÁFICAS}

Arbogast RT, Lecat GL, y Byrd RV. 1980. External morphology of some eggs of stored product moths (Lepidoptera: Pyralidae, Gelechiidae, Tineidae). Int. J.Insect Morphol. Embryol. 9: 165-177.

Chapman RF. 1982. The Insects: Structure and Function. 3nd Ed. Harvard University Press. Cambridge, Massachusetts EEUU. Pp. 392-393.

Chávez L y Añez N. 2003. Geometría de las células del exocorión de huevos de Rhodnius prolixus Stal, (Heteroptera: Reduviidae). Entomotrópica 18 (1): $1-5$.

Cruz-Reyes A y Camargo-Camargo B. 2001. Glosario de términos en parasitología y ciencias afines. Instituto de Biología. UNAM. México. ISSN 968-856-878-3.

Cummings MR y King RC. 1996. The cytology of the vitellogenic stages during oogenesis in Drosophila melanogaster. I. General staging characteristics. J. Morphol. 128: 427-442.

Dangles O, Carpio C, Barragán AR, Zeddam JL y Silvain JF. 2008. Temperature as a key driver of ecological sorting among invasive pest species in the tropical Andes. Ecological applications 18 (7): 1795-1809.

Deep DS y Rose HS. 2014. Study on the external morphology of the eggs of maize borer, Chilo partellus (Swinhoe). Journal of Entomology and Zoology Studies 4: 2

Freitas AVL y Brown KS. 2004. Phylogeny of the Nymphalidae (Lepidóptera). Systematic Biology 53: 363-383.

Gaino E, Piersanti M y Rebora M. 2008. Egg envelope synthesis and chorion modification after oviposition in the dragonfly Depressa (Odonata, Libellulidae). Tissue Cell 44: 317-324.

García-Barros E y Martin J. 1995. The eggs of European satyrine butterflies (Nymphalidae): external morphology and its use in systematics. Zool. J. Linn. Soc. 115: 73-115.

Gautam SG, Opit GP, Margosan D, Hoffmann D, Tebbets JS y Walse S. 2015. Comparative egg morphology and chorionic ultrastructure of key stored-product insects pests. Ann. Entomol. Soc. Am. 108: 43-56.

Gautam SG, Opit GP, Margosan D, Hoffmann D, Tebbets JS y Walse S. 2014. Egg morphology of key stored-product pests of the United States. Ann. Entomol. Soc. Am. 107: 1-10.

Hernández B, Flores A y Llorente J. 2014a. Morfología del corión en la Subfamilia Coliadinae (Lepidoptera: Pierida): Chorionic morphology in the Coliadinae Subfamily (Lepidoptera: Pieridae). Southwestern Entomologist 39(4): 853-886.

Hernández B, Flores Ay Llorente J. 2014b. Chorion morphology in the genus Ascia and Ganyra and its comparison with other proximate genera to Pierinae (Lepidoptera: Pieridae). Southwestern Entomologist 39 (1): 119-134.

Hinton HE. 1981. Biology of the insect egg, Tom I-III. Pergamon Press, Oxford.

Kawaguchi Y, Ichida M, Kusakabe T y Kogam K. 2007. Surface ultrastructure of the egg chorion of Eri silkworm, Samia ricini (Donovan) (Lepidoptera: Saturniidae). J. Entomol. 4: 68-81.

Kroschel J. 2002. Desafíos y oportunidades para el manejo de plagas en papa en países en desarrollo. Boletín del Centro Internacional de la Papa (CIP) División de Manejo de Cultivo, Agroecología MIP, Lima, Perú.

Kučerová Z, Hromádková J y Stejskal V. 2010. External egg morphology of common storedproduct pests from the families Anobiidae (Ptininae) and Dermestidae (Coleoptera). 10th International Working Conference on Stored Product Protection.

Llorente-Bousquets J y Castro G. 2007. Estudios en sistemática de Dismorphiinae (Lepidóptera: Pieridae) I: Morfología de huevos y su importancia taxonómica. Rev. Acad. Colomb. Cienc. 31(118): 145-164. ISSN 0370-3908.

Martínez C. 2015. Caracterización de la microestructura del corión del huevo de la polilla de la papa Symmetrischema tangolias y 
Tecia solanivora (Lepidóptera: Gelechiidae): Propuesta de un modelo estocástico para la identificación entre las dos especies. Disertación de Licenciatura, Pontificia Universidad Católica del Ecuador, Quito.

Mora-Criollo P, Rodríguez-Guerra A y Soria CA. 2013. Caracterización de la actividad amilásica presente en extractos larvarios de dos polillas plagas de la papa: Tecia solanivora y Symmetrischema tangolias. Rev. Ecuat. Med. Cienc. Biol. 34: 113127.

Outram I. 1967. Factors affecting the resistance of insect eggs to sulphuryl fluoride-I: the uptake of sulphuryl- ${ }^{35}$ S. fluoride by insect eggs. J. Stored Prod. Res. 3: 255-260.

Poprawa I y Rost MM. 2004. Structure and ultrastructure of the egg capsule of Thermobia domestica (Packard) (Insecta Zygentoma). Folia Biol 52(3-4): 185-190.

Rosciszewska E. 1996a. Diversification of the follicular cells in the panoistic ovary of the stone fly Perlodes intricata (Pictet, 1841) (Plecoptera: Perlodidae) during choriogenesis. Zool Poloniae 41: 89-102.

Rosciszewska E. 1996b. Egg capsule structure of the stonefly, Protonemura intricate (RIS, 1902) (Plecoptera: Nemuridae) Acta Biol. Cracov Ser Zool. 38: 41-49.

Sánchez-Otero J. 2015. Introducción a la estadística no paramétrica y al análisis multivariado. Giro Creativo. Quito, Ecuador.

Simiczyjew B. 1999. The ovary structure and oogenesis in Hydrometra stagnorum (Heteroptera: Hydrometridae). Acta Soc. Zool Bohem. 63: 187197.

Skudlik J, Poprawa I y Rost MM. 2005. The egg capsule of Spodoptera exiqua Hübner, 1808 (Insecta, Lepidoptera, Noctuidae): morphology and ultrastructure. Zoolog. Pol. 50: 25-31.

Sporleder M, Carhuapoma P y Kroschel J. 2016. Andean potatoe tuber moth, Symmetrischema tangolias (Gyen 1913). En: Kroschel, J., Mujica, N., Carhuapoma, P., Sporleder, M. (Eds). Pest distribution and risk atlas for Africa. Potential global and regional distribution and abundance of agricultural and horticultural pests and associated biocontrol agents and their current and future climates. Lima (Perú). International potato center (CIP).pgs, 39-53.

Suludere Z. 1988. Studies on the external morphology of the eggs of Melitaea species (Satyridae: Lepidoptera). Commun. Fac. Sci. Univ. Ank. Ser. C 5: 73-84.

Tuft HP. 1950. The structure of the insect egg-shell in relation to the respiration of the embryo. Exp. Biol. 26: 327-334.

Van Trees HL, Bell KL y Tian Z. 2013. Detection, Estimation and Modulation theory, Part I., 2nd Ed., John Wiley \& Sons Inc.

Visciarelli E, Ferrero A y Costamanga SR. 2004. Aspectos exocoriales de huevos de Triatoma patagónica del Ponte, 1929 por microscopía electrónica de barrido. Entomol. Vect. 11(4): 653668. ISSN 0328-0381. 